Supporting Information

\title{
On the Nature of Active Sites of $\mathrm{VO}_{\mathrm{x}} / \mathrm{Al}_{2} \mathrm{O}_{3}$
}

\section{Catalysts for Propane Dehydrogenation}

Gang Liu ${ }^{1}$, Zhi-Jian Zhao ${ }^{1}$, Tengfang Wu, Liang Zeng, and Jinlong Gong*

Key Laboratory for Green Chemical Technology of Ministry of Education, School of

Chemical Engineering and Technology, Tianjin University, Collaborative Innovation

Center of Chemical Science and Engineering, Tianjin 300072, China

[1] These authors contributed equally to this work.

[*] Corresponding author: jlgong@tju.edu.cn 


\section{Table of Contents}

1. UV-vis spectra of $\mathrm{VO}_{\mathrm{x}} / \mathrm{Al}_{2} \mathrm{O}_{3}$ catalysts and calculated edge energy.

2. EPR profile of the reduced $1 \mathrm{~V} / \mathrm{Al}$ catalyst.

3. DRFITS spectra of propane adsorption and evacuation at $30{ }^{\circ} \mathrm{C}$ on the $12 \mathrm{~V} / \mathrm{Al}$ catalyst.

4. IR spectra of the spent $12 \mathrm{~V} / \mathrm{Al}$ catalyst.

5. IR spectra of acetone and formic acid adsorbed over the $12 \mathrm{~V} / \mathrm{Al}$ catalyst.

6. Deconvolution of the fit of the $\mathrm{V} \mathrm{2} \mathrm{p}_{2 / 3}$ signal for the $6 \mathrm{~V} / \mathrm{Al}$ catalyst after one-hour reaction.

7. Mass transfer analysis

8. Details on DFT parameters. 

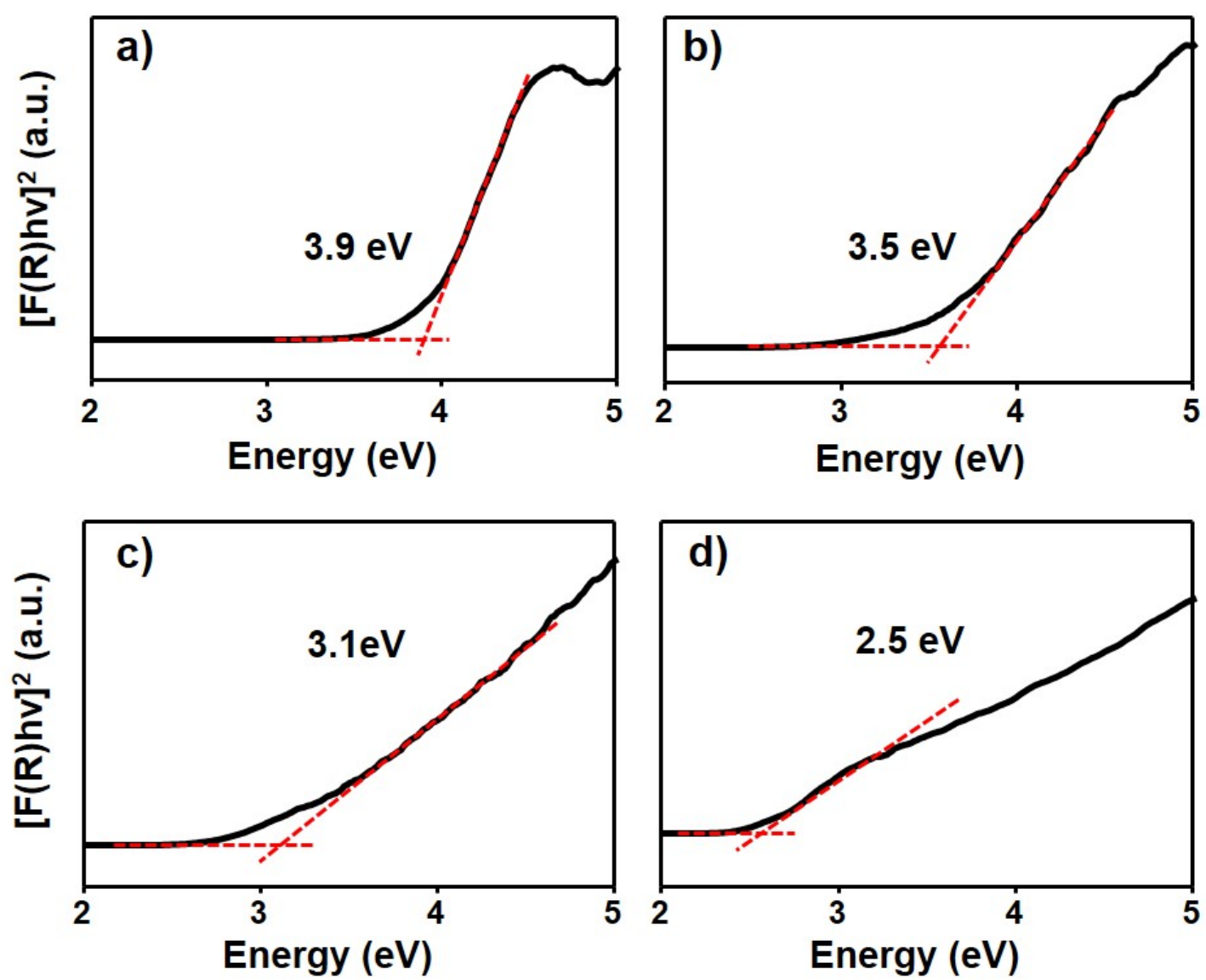

Figure S1. UV-vis spectra and calculated edge energy of the a) $1 \mathrm{~V} / \mathrm{Al}$, b) $6 \mathrm{~V} / \mathrm{Al}$, c) $12 \mathrm{~V} / \mathrm{Al}, \mathrm{d}) 20 \mathrm{~V} / \mathrm{Al}$ catalysts. 


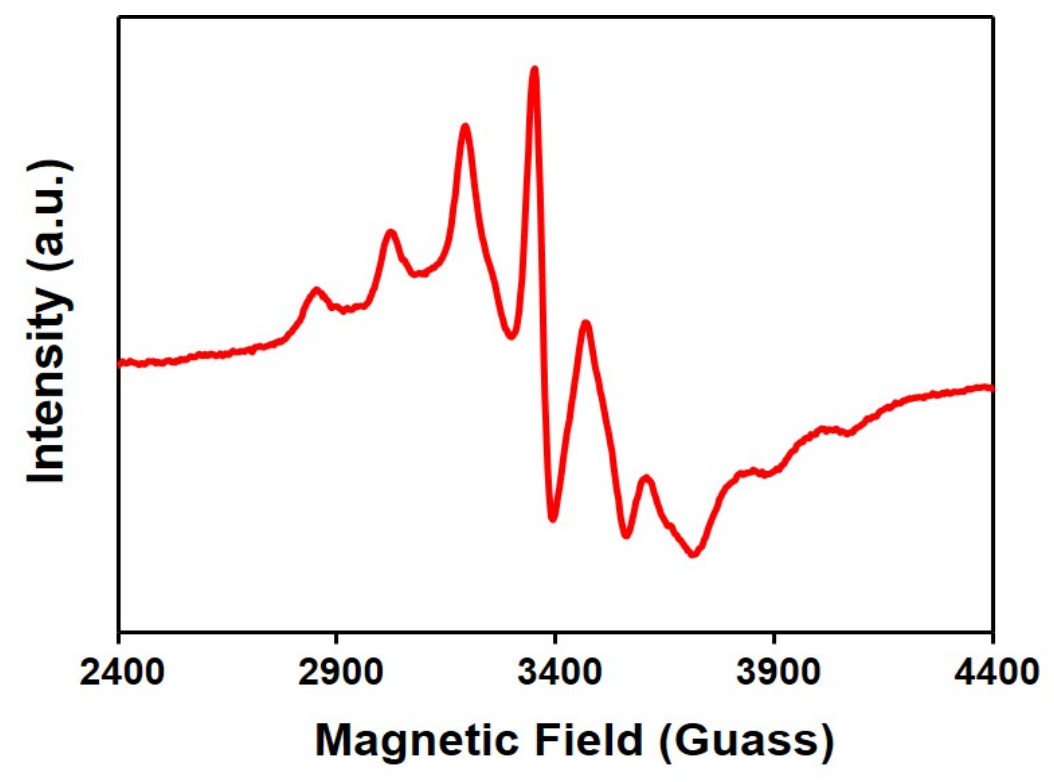

Figure S2. EPR profile of the reduced $1 \mathrm{~V} / \mathrm{Al}$ catalyst. 


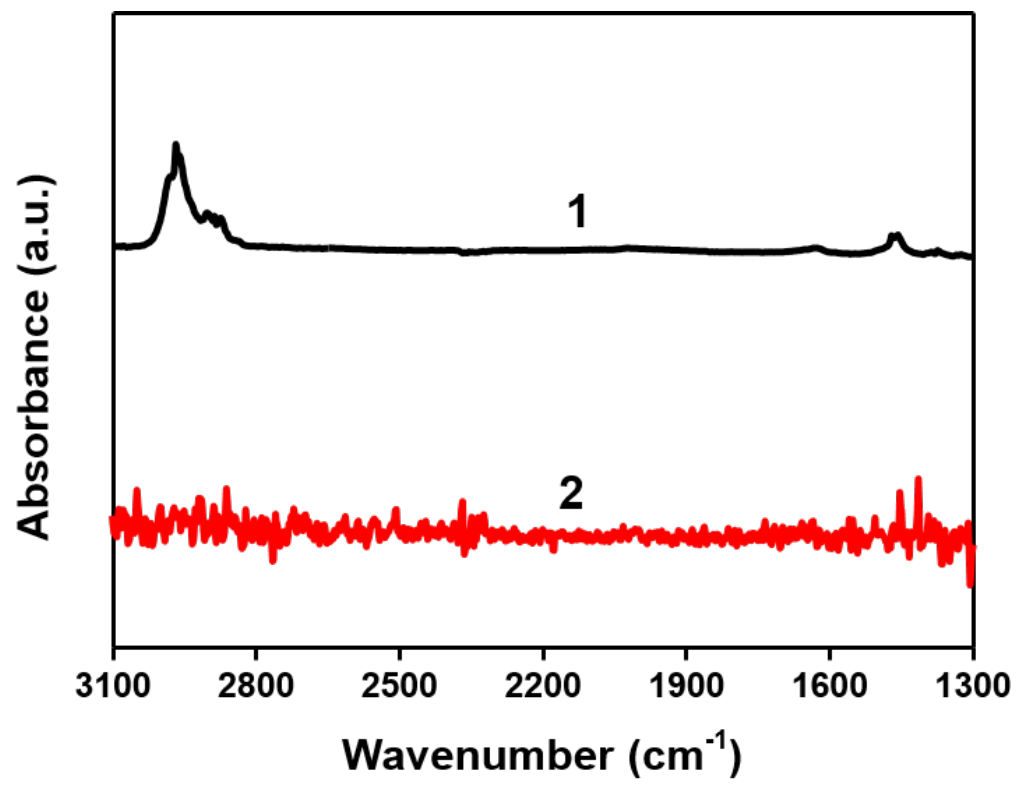

Figure S3. DRFITS spectra of 1) propane adsorption at $30^{\circ} \mathrm{C}, 2$ ) and evacuation at 30 ${ }^{\circ} \mathrm{C}$ on the $12 \mathrm{~V} / \mathrm{Al}$ catalyst. 


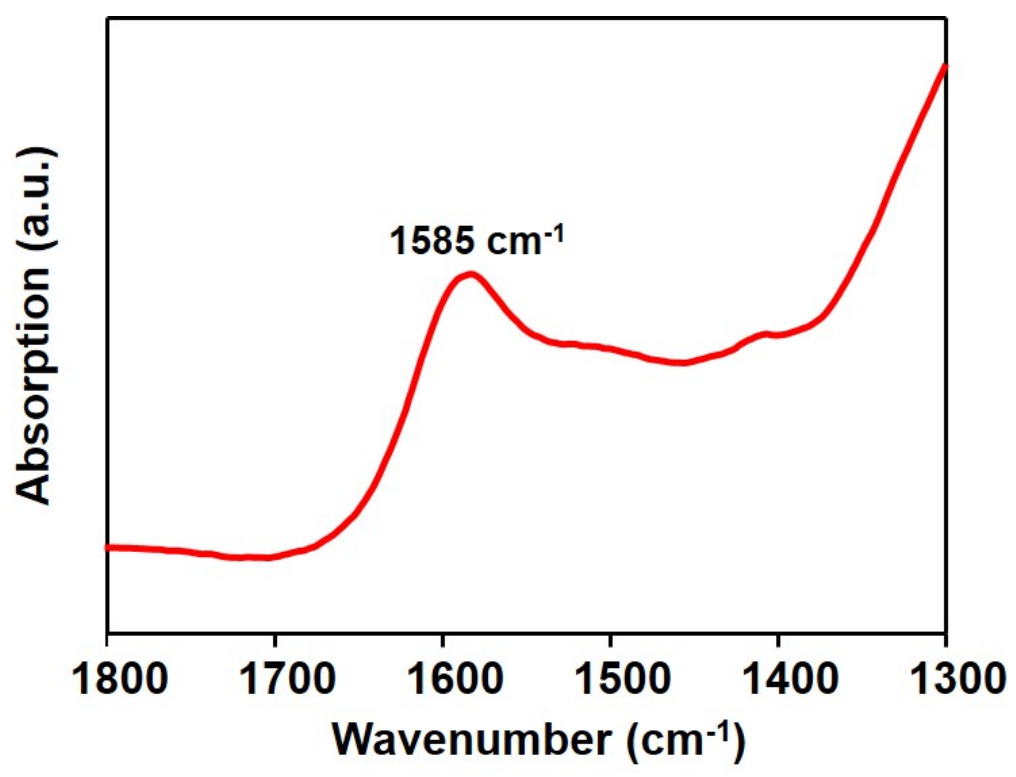

Figure S4. IR spectra of the spent $12 \mathrm{~V} / \mathrm{Al}$ catalyst. 

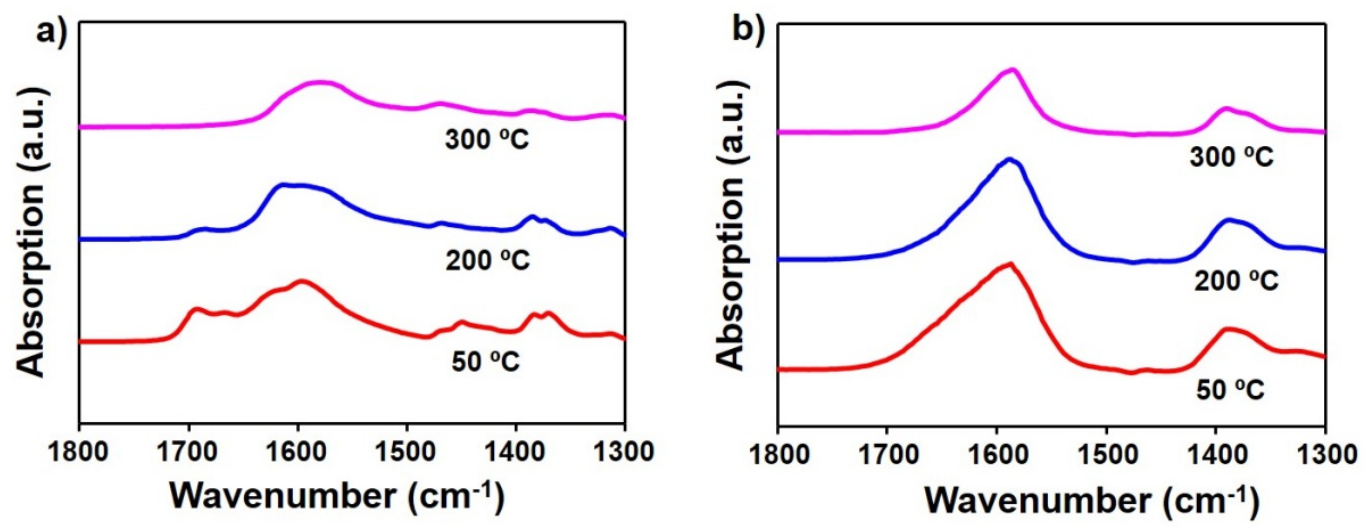

Figure S5. IR spectra of a) acetone and b) formic acid adsorbed over the 12V/Al catalyst. 


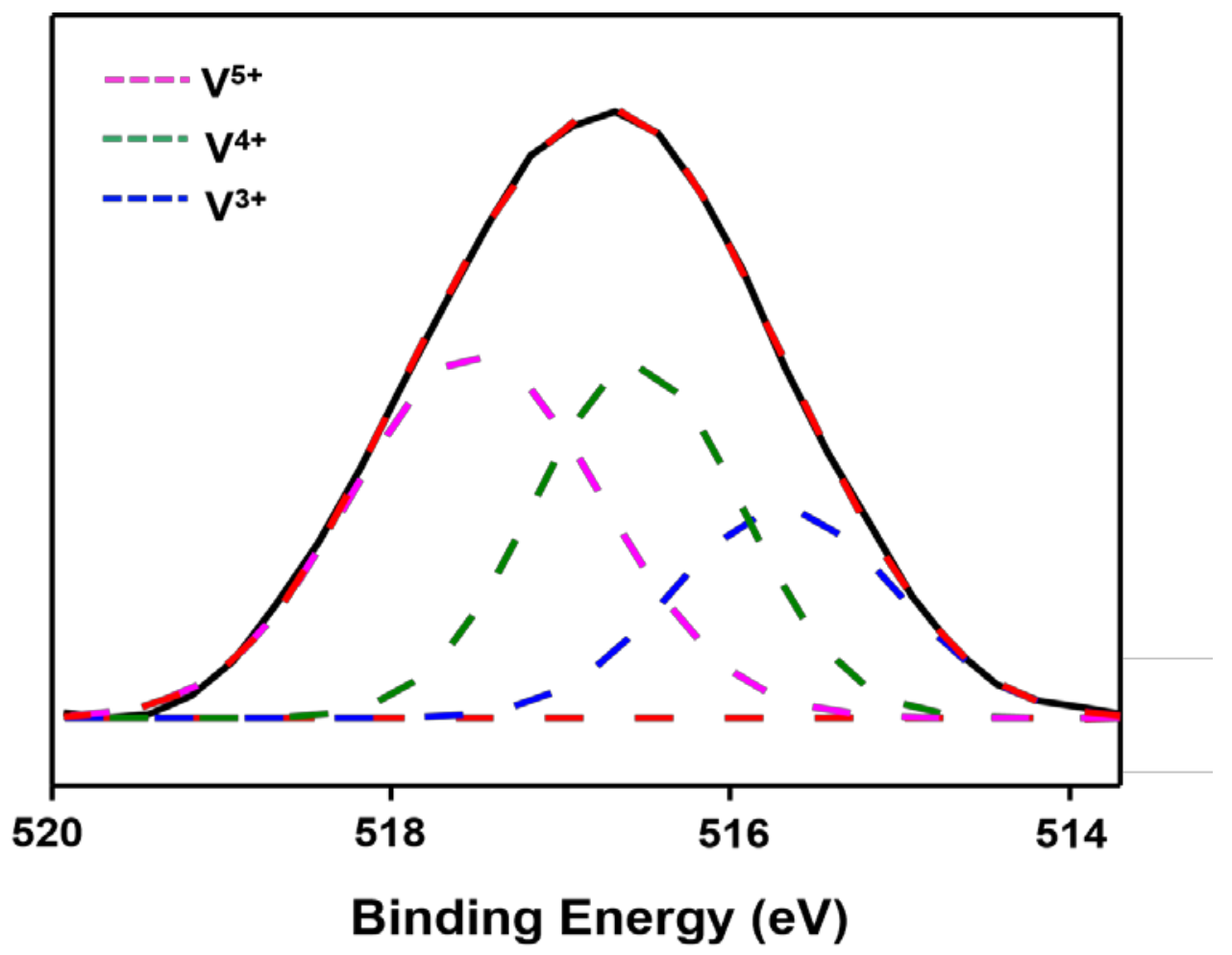

Figure S6. Deconvolution of the fit of the $\mathrm{V} \mathrm{2} \mathrm{p}_{2 / 3}$ signal for the $6 \mathrm{~V} / \mathrm{Al}$ catalyst after one-hour reaction. 


\section{Mass transfer analysis}

Mass transport calculations were carried out for the highest rates over the 12V/Al sample via the Weisz-Prater criterion. ${ }^{1}$

$C_{W P}=\frac{-r_{A}^{\prime} \rho_{c} R^{2}}{D_{e} C_{A S}}<1$

$-r_{A}^{\prime}$ : observed reaction rate, $\mathrm{kmol} / \mathrm{kg}_{\mathrm{cat}} \mathrm{s}$;

$R$ : catalyst particle radius, $\mathrm{m}$;

$\rho_{c}$ : solid catalyst density, $\mathrm{kg} / \mathrm{m}^{3}$;

$D_{e}$ : effective gas-phase diffusivity, $\mathrm{m}^{2} / \mathrm{s}$

$$
=\frac{D_{A B} \phi_{p} \sigma_{c}}{\tau} \text { where }
$$

$D_{A B}$ : gas-phase diffusivity $\mathrm{m}^{2} / \mathrm{s} ;{ }^{\varphi_{p}}$ : pellet porosity; ${ }^{\sigma_{c}}$ : constriction factor; ${ }^{\tau}$ : tortuosity;

$C_{A S}$ : gas concentration of A at the catalyst surface, $\mathrm{kmol} / \mathrm{m}^{3}$;

In this study:

$-r_{A}^{\prime}=4.53 \times 10^{-7} \mathrm{kmol}^{\prime} \mathrm{kg}_{\text {cat }} \cdot \mathrm{S}$

$\rho_{c}=362 \mathrm{~kg} / \mathrm{m}^{3}$

$R=3.8 \times 10^{-4} \mathrm{~m}$

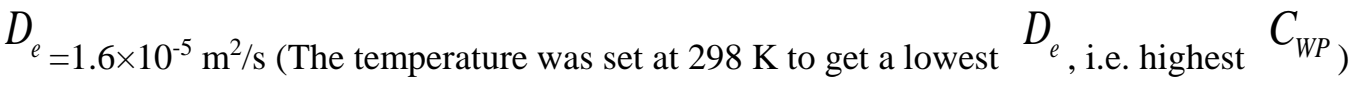

$C_{A S}=1.25 \times 10^{-2} \mathrm{kmol} / \mathrm{m}^{3}$

Then, $C_{W P}=1.2 \times 10^{-4}<1$, thus the internal diffusion limitations can be ignored. Therefore, the decreased apparent activation energy observed in this study must be related to the variation of active sites. 


\section{Details on DFT parameters}

DFT calculations were performed with the plane-wave based Vienna ab initio simulation package VASP ${ }^{2,3}$ with PBE exchange-correlation functional. ${ }^{4}$ The $(2 \mathrm{x} 2)$ $\alpha-\mathrm{Al}_{2} \mathrm{O}_{3}(0001)$ was represented by periodic slab models of six complete $\mathrm{Al}-\mathrm{O}-\mathrm{Al}$ tri-layer thickness. One surface $\mathrm{Al}$ atom was replaced by $\mathrm{V}$ atom and the $\mathrm{C}_{3} \mathrm{H}_{5}$ species was attached to the $\mathrm{V}$ atom. The valence wave functions were expanded in a plane-wave basis with a cutoff energy of $400 \mathrm{eV}$, while the interaction between the atomic cores and the electrons was described by the projector augmented wave (PAW) method. ${ }^{5,6}$ A Monkhorst-Pack mesh ${ }^{7}$ of $3 \times 3 \times 1$ was used to sample the Brillouin. All atoms were allowed to relax during geometry optimizations, together with the adsorbate, until the force on each atom was less than $2 \times 10^{-4} \mathrm{eV} / \mathrm{pm}$. To obtain the vibrational frequencies, a normal mode analysis, wherein the elements of the Hessian were approximated as finite differences of gradients, is carried out, with positive and negative displacements of $0.015 \AA$. In addition, we believe the influence by the different substrate $\left(\alpha-\mathrm{Al}_{2} \mathrm{O}_{3}\right.$ in cal. vs. $\gamma-\mathrm{Al}_{2} \mathrm{O}_{3}$ in exp.) is minor, since the $\mathrm{C}-\mathrm{H}$ vibrations is not strongly mixed with the substrate. Our calculated $\mathrm{C}=\mathrm{C}$ stretch mode $\left(1566 \mathrm{~cm}^{-1}\right)$ is even very close to the similar species, $\eta-\mathrm{CHCH}_{2}$, on $\operatorname{Pt}(111)$ (1547 $\left.\mathrm{cm}^{-1}\right) .^{8}$ 


\section{References}

1. Oyama S. T., Zhang X., Lu J., Gu Y., Fujitani T. J. Catal. 2008, 257, 1-4.

2. Kresse, G.; Hafner, J. Phys. Rev. B 1994, 49, 14251-14269.

3. Kresse, G.; Furthmüller, J. Comp. Mat. Sci. 1996, 6, 15-50.

4. Perdew, J. P.; Burke, K.; Ernzerhof, M. Phys. Rev. Lett. 1996, 77 (18), 3865-3868.

5. Blöchl, P. E. Phys. Rev. B 1994, 50, 17953-17979.

6. Kresse, G.; Joubert, D. Phys. Rev. B 1999, 59, 1758-1775.

7. Monkhorst, H. J.; Pack, J. D. Phys. Rev. B 1976, 13, 5188-5192.

8. Zhao, Z.-J.; Moskaleva, L. V.; Aleksandrov, H. A.; Basaran, D.; Rösch, N. J. Phys. Chem. C 2010, 114, 12190-12201. 